\title{
Impacto Económico de Laringotraquítis Infecciosa Aviar en una Granja de Pollos de Carne en Lima, Perú
}

\author{
ECONOMIC Impact OF AVIAN INFECTIOUS LARYNGotracheItIS IN A BroILERS FARM IN \\ Lima, Perú
}

\author{
Lupe López L. ${ }^{1}$, Eliana Icochea D. ${ }^{1,4}$, Pablo Reyna S. ${ }^{1}$, Carlos Angulo J. ${ }^{2}$, \\ Raúl Zegarra V. ${ }^{3}$
}

\section{Resumen}

El objetivo de este estudio fue cuantificar el impacto económico de la laringotraqueítis infecciosa aviar (LTI) en el rendimiento productivo y económico de pollos de engorde. Se evaluaron dos campañas consecutivas de 35000 pollos criados hasta los 49 días de edad, donde la primera campaña estuvo afectada por LTI. Se realizó una encuesta para recopilar información sobre datos productivos, bioseguridad y costos de producción por campaña, así como los gastos adicionales que demandó el control de la enfermedad. El impacto económico se evaluó mediante la comparación de los datos de ambas campañas usando un modelo de distribución estocástica con el programa de análisis de riesgos @ Risk 5.1 ${ }^{\circledR}$. Además, se hizo un análisis estadístico para determinar diferencias entre datos productivos de cada campaña. En la campaña con LTI se incrementó la mortalidad en $3.51 \%$ y el índice de conversión en $16.8 \%$, y la eficiencia productiva se redujo en $27.3 \%$ en comparación con la campaña sin LTI. Las pérdidas totales por la enfermedad sumaron $\mathrm{S} / .88,805$. El análisis de riesgos mostró un rango de pérdidas de S/. 28,800.00 hasta S/. 149,300.00 con un 95\% de probabilidad, para los 35,000 pollos de engorde con LTI.

Palabras clave: laringotraqueítis infecciosa aviar, pollos de engorde, impacto económico

\footnotetext{
${ }^{1}$ Laboratorio de Patología Aviar, ${ }^{2}$ Laboratorio de Medicina Veterinaria Preventiva, Facultad de Medicina Veterinaria, Universidad Nacional Mayor de San Marcos, Lima, Perú

${ }^{3}$ Servicio Nacional de Sanidad Agraria, Lima, Perú

${ }^{4}$ E-mail: eliana.icochea@gmail.com
}

Recibido: 10 de setiembre de 2014

Aceptado para publicación: 20 de mayo de 2015 
The aim of this study was to quantify the economic impact of avian infectious laryngotracheitis (ILT) on productive and economic performance of broilers. Two batches of 35000 broilers each up to 49 days of age were evaluated. The first batch was affected for ILT while the other was not affected. A survey was conducted to collect information about productive data, biosecurity and production cost per batch, as well as additional costs related to the control of the disease. The economic impact was evaluated by comparing data from both batches using a stochastic distribution model with the risk analysis program $@$ Risk 5.1 ${ }^{\circledR}$. Besides, a statistical analysis was done to determine difference between productive data of the two batches. In the one with ILT the mortality increased by $3.51 \%$ and feed conversion rate by $16.8 \%$, while the productive efficiency decreased by $27.3 \%$ compared with the batch without ILT. The total economical loses due the disease were S/. 88,805. The risk analysis showed a range of losses from S/. 28,800.00 to S/. 149,300.00 for the 35000 broilers with ILT.

Key words: avian infectious laryngotracheitis, broilers, economic impact

\section{INTRODUCCIÓN}

La laringotraqueítis infecciosa de las aves (LTI) es una enfermedad respiratoria altamente contagiosa, de distribución mundial y ocurrencia cíclica en zonas endémicas, principalmente en áreas de alta densidad de producción. Los pollos son susceptibles a la infección, aunque también puede afectar a faisanes, perdices y pavos (Guy y García, 2008). En el Perú, la enfermedad fue reportada por primera vez en agosto de 2008 en gallos de pelea (JR Zegarra, comunicación personal). Está considerada dentro de las Enfermedades de Declaración Obligatoria por la Organización Mundial de Sanidad Animal (OIE). La presencia de la enfermedad constituye un serio impedimento para el comercio internacional de productos y subproductos avícolas (OIE, 2005).

LTI es causada por un virus neumotrópico, miembro de la familia Herpesviridae, el cual se transmite por contacto directo, aerosoles y fómites contaminados con secreciones de aves afectadas, donde el hombre participa en su diseminación entre granjas (Sellers et al., 2004). La diseminación del virus dentro de un galpón es rápi- da y entre galpones es lenta; asimismo, no se ha demostrado la transmisión vía ovo (Cover, 1996; Dufour-Zavala, 2008).

En la prevención y control de la LTI, es indispensable la cuarentena e higiene de la granja afectada, así como evitar el movimiento de personal, alimento, equipo y aves potencialmente contaminadas. También se deben implementar medidas de control para roedores y perros y reconocer el riesgo de contaminación de enfermedades persistentes que constituyen las parvadas de traspatio y aves de exhibición (Guy y García, 2008).

La importancia de esta enfermedad radica en las al tas pérdidas económicas causadas por la al ta mortalidad y morbilidad, seve ra disminución en la producción de huevos y en el desempeño productivo de las aves. Ade más, hay que considerar los gastos por la implementación de procedimientos de control de la enfermedad. La LTI para pollos de engorde en la forma epizoótica presenta una morbilidad de 90 a $100 \%$ y mortalidad de 5 a $70 \%$, mientras que las formas enzoóticas pre sentan una morbilidad de $5 \%$ y una mortalidad de 0.1 a $2 \%$ (Guy y Baugust, 2003). En ambas formas, se observa disminución de los parámetros productivos y altos costos por 
medicación, los cuales se incrementan con las infecciones secundarias (Alexander y Nagy, 1997; Humberd et al., 2002; Manathan, 2006; Chacón, 2008).

El objetivo de este estudio fue cuantificar el impacto económico de la laringotraqueítis infecciosa aviar sobre el rendimiento productivo y económico en una granja de pollos de engorde en el departamento de Lima.

\section{Materiales y Métodos}

\section{Lugar de Estudio}

El estudio se realizó en una granja de pollos de engorde ubicada en la Cooperativa Agropecuaria de las Vertientes, en el distrito de Villa el Salvador, departamento de Lima, Perú. La granja constaba de cuatro galpones (dos de hembras y dos de machos), donde las aves son criadas hasta los 49 días de edad. Se trabajó con dos campañas de 35000 pollos de la línea Cobb Vantres, la primera, afectada por LTI, se llevó a cabo durante marzo y abril de 2010, en tanto que la segunda campaña, sin LTI, fue de junio a julio de 2010.

\section{Recolección de Datos}

Se recolectó información mediante visitas a la granja, empleando una encuesta adecuada a partir de la «Ficha modificada de información y compromiso de gestión para el fortalecimiento del sistema sanitario avícola» elaborada por el Servicio Nacional de Sanidad Agraria (SENASA), así como de los registros de producción de la granja. Además, se tomaron datos de bioseguridad de las fichas de trabajo de la granja.

\section{Evaluación de Parámetros Productivos}

En ambas campañas se evaluó el índice de conversión alimenticia (ICA), según la fórmula: Alimento consumido / Peso vivo; el índice de eficiencia productiva (IEP), según la fórmula: (Viabilidad x Ganancia diaria de peso x 100) / ICA, y la tasa de mortalidad; a los cuales se determinó su distribución normal mediante la prueba de Shapiro Wilk. Además, se realizó un análisis de varianza para establecer diferencias estadísticas entre grupos, mediante el programa estadístico Stata 12.0 .

\section{Evaluación del Impacto Económico}

La evaluación del impacto económico de LTI sobre la granja de pollos de engorde se realizó con base en la sumatoria de las pérdidas producidas y los gastos implementados para controlar la enfermedad, teniendo como modelo control la campaña no afectada con LTI. Se realizó un modelo de distribución estocástica con el programa para análisis de riesgo @ Risk 5.1 ${ }^{\circledR}$ (10 000 iteracciones), con la intención de obtener una curva de distribución de las ganancias económicas para cada campaña y la diferencia entre ellas.

\section{Evaluación de la Bioseguridad}

El nivel de bioseguridad de la granja fue evaluado mediante la «Hoja modificada de cálculo de puntaje de bioseguridad para granjas avícolas» del SENASA. Además, se utilizaron los registros de producción y se cuantificaron los valores económicos de cada campaña del estudio, considerando gastos por diagnóstico, medicamentos y desinfectantes.

\section{Resultados y Discusión}

El comportamiento productivo de las aves, medido en términos de ICA, IEP y mortalidad fue significativamente afectado por la LTI ( $\mathrm{p}<0.05$; Cuadro 1). Los resultados confirman el impacto negativo que causa la LTI en la producción de pollos de engorde, generando pérdidas económicas por disminución de los parámetros productivos, aumento de la mortalidad y consumo de medicamentos (Mclnerney, 1994; Alexander y Nagy, 1997; Chacón, 2008). 
Cuadro 1. Índice de conversión alimenticia (ICA), índice de eficiencia productiva (IEP) y mortalidad (\%) en pollos de engorde de las campañas con y sin laringotraqueítis infecciosa aviar (LTI) hasta los 49 días de edad (Lima, 2010)

\begin{tabular}{lclccc}
\hline Campaña & Galpón & Sexo & ICA & IEP & $\begin{array}{c}\text { Mortalidad } \\
(\%)\end{array}$ \\
\hline Con LTI & I & Macho & 2.37 & 219.36 & 8.09 \\
& II & Hembra & 2.40 & 179.19 & 7.20 \\
& III & Macho & 1.94 & 269.89 & 7.47 \\
& IV & Hembra & 2.41 & 176.67 & 8.05 \\
\cline { 2 - 6 } & Promedio & & $2.28^{\mathrm{a}}$ & $211.28^{\mathrm{a}}$ & $7.69^{\mathrm{a}}$ \\
\hline \multirow{3}{*}{ in LTI } & I & Macho & 1.86 & 320.57 & 4.79 \\
& II & Hembra & 2.06 & 256.21 & 3.73 \\
& III & Macho & 1.82 & 327.40 & 4.80 \\
& IV & Hembra & 2.05 & 258.06 & 3.46 \\
\cline { 2 - 6 } & Promedio & & $1.95^{\mathrm{b}}$ & $290.56^{\mathrm{b}}$ & $4.20^{\mathrm{b}}$ \\
\hline
\end{tabular}

${ }^{a, b}$ Superíndices diferentes dentro de columnas indican diferencia estadística $(p \varangle 0.05$ )

Cuadro 2. Kilogramos de pollo producidos en campañas con y sin laringotraqueítis infecciosa aviar (LTI) (Lima, 2010)

\begin{tabular}{lccc}
\hline \multirow{2}{*}{ Campaña } & \multicolumn{2}{c}{ Kilogramo de pollo producido } & \multirow{2}{*}{$\begin{array}{c}\text { Merma } \\
(\%)\end{array}$} \\
\cline { 2 - 3 } & Sin LTI & Con LTI & 13.6 \\
Machos & 50,711 & 43,824 & 19.6 \\
\hline Hembras & 45,110 & 36,286 & 16.4 \\
\hline Total & 95,821 & 80,109 & \\
\hline
\end{tabular}

Los mortalidad observada se encuentra en el límite inferior del rango de una presentación epizoótica de la enfermedad (5 a 70\%), según lo describen Guy y Baugust (2003); sin embargo, fue mayor que las reportadas por el mismo autor para las formas enzoóticas leves de la enfermedad (0.1 a $2 \%)$.
La producción de pollo (en kilogramos) en la campaña con LTI, tanto de machos como de hembras, disminuyó en un $16.4 \%$ en comparación con la campaña sin LTI; siendo las hembras las que perdieron mayor peso corporal (19.6\%) en comparación con el $13.6 \%$ de los machos (Cuadro 2). 
Cuadro 3. Comparación del rendimiento económico de pollos de engorde en campañas con y sin laringotraqueítis infecciosa aviar (LTI)

\begin{tabular}{|c|c|c|c|c|}
\hline Campaña & Con LTI & $\%$ & Sin LTI & $\%$ \\
\hline \multicolumn{5}{|l|}{ Egresos } \\
\hline \multicolumn{5}{|l|}{ Costos fijos (S/.) } \\
\hline Depreciación de construcciones & $3,000.00$ & 0.92 & $3,000.00$ & 0.97 \\
\hline Dep. de instalaciones & $4,000.00$ & 1.22 & $4,000.00$ & 1.30 \\
\hline Dep. de equipos e implementos & $2,800.00$ & 0.85 & $2,800.00$ & 0.91 \\
\hline Dep. de motores & 500.00 & 0.15 & 500.00 & 0.16 \\
\hline Mantenimiento de construcciones & $2,500.00$ & 0.76 & $2,500.00$ & 0.82 \\
\hline Mant. de instalaciones & $2,000.00$ & 0.61 & $2,000.00$ & 0.65 \\
\hline Mant. de equipos e implementos & $3,000.00$ & 0.92 & $3,000.00$ & 0.97 \\
\hline Mantenimiento de motores & $4,000.00$ & 1.22 & $4,000.00$ & 1.30 \\
\hline Servicios básicos & 400.00 & 0.12 & 400.00 & 0.13 \\
\hline Personal fijo & $4,000.00$ & 1.22 & $4,000.00$ & 1.30 \\
\hline Materiales y suministros & $16,777.86$ & 5.12 & $16,777.86$ & 5.47 \\
\hline Sub total costos fijo & $42,977.86$ & 13.12 & $42,977.86$ & 14.01 \\
\hline \multicolumn{5}{|l|}{ Costos variables $(\mathrm{S} /)}$. \\
\hline Aves & $46,667.67$ & 14.25 & $44,333.33$ & 14.46 \\
\hline Alimento $^{1}$ & $232,791.47$ & 71.07 & $218,512.00$ & 71.25 \\
\hline Desinfectantes & 787.89 & 0.24 & 375.98 & 0.12 \\
\hline Medicamentos & $3,350.11$ & 1.02 & 420.18 & 0.14 \\
\hline Pruebas auxiliares & 998.00 & 0.30 & 60.00 & 0.02 \\
\hline Sub total costos variables & $284,594.14$ & 86.88 & $263,701.50$ & 85.99 \\
\hline $\begin{array}{l}\text { Total (costos fijos + costos } \\
\text { variables) }\end{array}$ & $327,572.26$ & & $306,679.36$ & \\
\hline \multicolumn{5}{|l|}{ Ingresos (S/.) } \\
\hline Venta total de $\mathrm{kg}$ pollo producido ${ }^{2}$ & $340,896.17$ & & $408,809.41$ & \\
\hline Ganancia (S/.) & $13,324.17$ & 3.91 & $102,130.05$ & 24.98 \\
\hline Costos/kg de pollo (S/.) & 4.09 & & 3.20 & \\
\hline
\end{tabular}

El aumento de los costos variables de la campaña con LTI dió como resultado un costo adicional de S/. 0.89 por kilogramo de pollo, equivalente a $27.76 \%$ más que la campaña sin LTI (Cuadro 3). La diferencia de ganancias entre ambas campañas se calculó en S/.88,805.88.
Mediante el programa de análisis de riesgo @Risk 5.1 ${ }^{\circledR}$, se calculó que en la campaña con LTI, el rango de ganancias fue de S/. $-27,000$ a S/. 56,000, en comparación con la campaña sin LTI donde el rango fue de S/. $60,400.00$ a S/. 147,800.00 (Fig. 1). Para la ganancia entre ambas campañas se determi- 

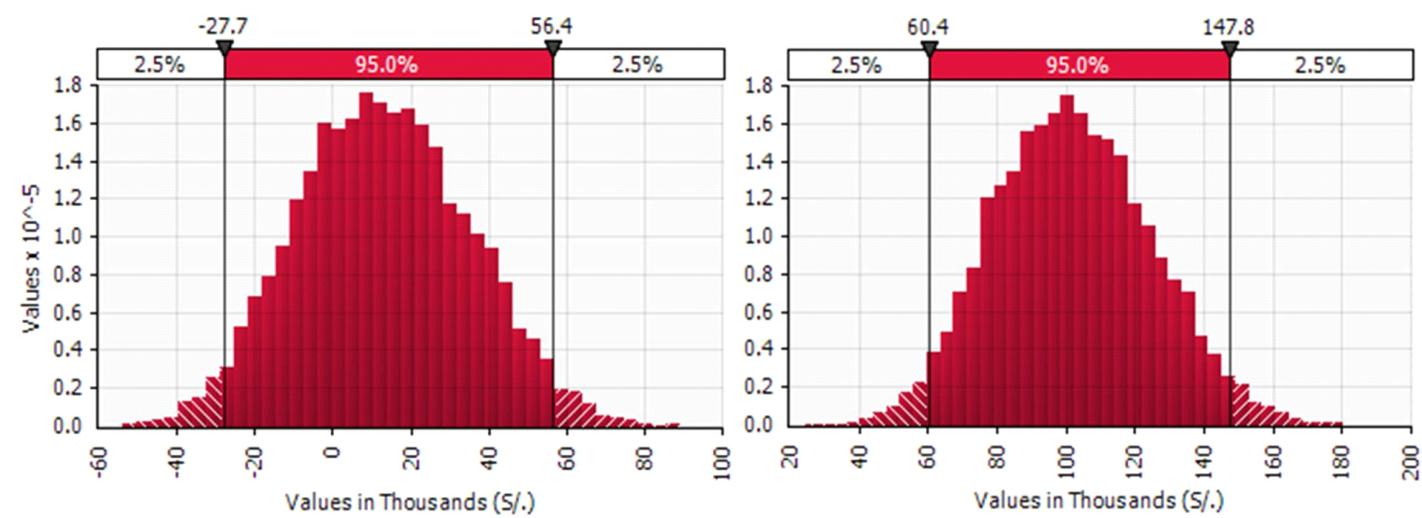

Figura 1. Curva de distribución de ganancias (S/.) con pollos de engorde al final de la campaña con (izquierda) y sin (derecha) laringotraqueítis infecciosa aviar (LTI) (Lima, 2010)

nó un rango de $\mathrm{S} / .28,800.00$ y S/. 149,300.00 por los 35000 pollos de engorde.

El nivel de bioseguridad de la granja durante la campaña con LTI presentó un nivel considerado «Observable $»^{5}$ y para la campaña sin LTI se consideró en un nivel de «Riesgo mínimo». Estos hallazgos corroboran que la ineficiente bioseguridad es un factor predominante para iniciar los brotes de LTI en pollos de engorde (Dufour-Zavala, 2008).

\section{Conclusión}

La laringotraqueítis infecciosa aviar causó un impacto económico negativo en la granja evaluada. La campaña afectada por la enfermedad incrementó en $27.76 \%$ el costo de producción del kilogramo de pollo de engorde, incrementó la mortalidad en $3.51 \%$, redujo un $27.3 \%$ el índice de eficiencia productiva y aumentó el índice de conversión alimenticia en $16.8 \%$, en comparación con la campaña sin la enfermedad.

\section{Literatura Citada}

1. Alexander H, Nagy É. 1997. Polymerase chain reaction to detect infectious laryngotracheitis virus in conjunctival swabs from experimentally infected chickens. Avian Dis 41: 646-653.

2. Cover MS. 1996. The early history of infectious laryngotracheitis. Avian Dis 40: 494-500. doi: 10.2307/1592256

3. Chacón JL. 2008. Epidemiología molecular do vírus da laringotraqueíte infecciosa isolados de surtos em poedeiras comerciais no Estado de São Paulo. Tesis de Doctorado. Brasil: Universidade de São Paulo. 110 p.

4. Dufour-Zavala L. 2008. Epizootiology of infectious laryngotracheitis and presentation of an industry control program. Avian Dis 52: 1-7. doi: $10.1637 /$ 8018-051007-Review

5. Guy JS, Baugust TJ. 2003. Laryngotracheitis. In: Saif YM (ed). Diseases of poultry. $11^{\text {th }}$ ed. Iowa, USA: Iowa State Press. p 121-133.

\footnotetext{
${ }^{5}$ Falta de aislamiento de centros poblados y de carreteras, falta de garita de control, no desinfección de vehículos, falta de duchas para el personal y visitas, y ausencia de cambio de ropa para el ingreso a granja
} 
6. Guy JS, García M. 2008. Laryngotracheitis. In: Saif YM, Barnes HJ, Glisson JR, Fadly AM, Mc Dougald LR, Swayne DE (eds). Diseases of poultry. $12^{\text {th }}$ ed. Iowa, US A: Iowa State University Press. p 137-152.

7. Humberd J, Garcia M, Riblet SM, Resurrección RS, Brown TP. 2002. Detection of infectious laryngotracheitis virus in formalin-fixed, paraffinembedded tissues by nested polymerase chain reaction. Avian Dis 46: 64-74.

8. Manathan $P$. 2006. Infectious laryngotracheitis disease situation and control measures. Facsheet 155. Canada: Poultry Industry Council. 4 p.
9. Mclnerney J. 1994. ¿Cuánto cuesta la enfermedad? Ind Avícola 2: 12-15.

10. [OIE] Organización Mundial de Sanidad Animal. 2005. Antigua clasificación de enfermedades de declaración obligatoria a la OIE. [Internet]. Disponible en: http://www.oie.int/es/sanidad-animal-en-el-mundo/el-sistema-mundial-deinformacion-sanitaria/antiguaclasificacion-de-enfermedades-dedeclaracion-obligatoria-a-la-oie-lista-a/

11. Sellers HS, Garcia M, Glisson JR, Brown TP, Sander JS, Guy JS. 2004. Mild infectious laryngotracheitis in broilers in the Southeast. Avian Dis 48: 430-436. doi: 10.1637/7129 